Osservazioni meridiane fitte nell' J. R. Osservatorio di Padova.

Osservazioni meridiane di Giova.

\begin{tabular}{|c|c|c|c|}
\hline \multicolumn{2}{|c|}{1855} & J. M. di & Padova \\
\hline Agosto & 5 & $12^{l} 39^{\prime}$ & $24^{\prime \prime} 1$ \\
\hline & 6 & 1235 & 2,7 \\
\hline & 7 & 1230 & 41,4 \\
\hline & 17 & 1146 & 43,2 \\
\hline & $2 \cdot 2$ & 1124 & 34,0 \\
\hline & 23 & 1120 & 7,6 \\
\hline & 24 & 1115 & 41,3 \\
\hline & 25 & 1111 & 14,6 \\
\hline & 26 & 116 & 48,1 \\
\hline & 27 & 112 & 21,7 \\
\hline & 28 & $10 \quad 57$ & 55,3 \\
\hline & 29 & 1053 & 28,4 \\
\hline & 30 & 1049 & 2,1 \\
\hline Settembre & 4 & 1027 & 49,9 \\
\hline & 7 & 1013 & 32,4 \\
\hline & 11 & 955 & 52,7 \\
\hline
\end{tabular}

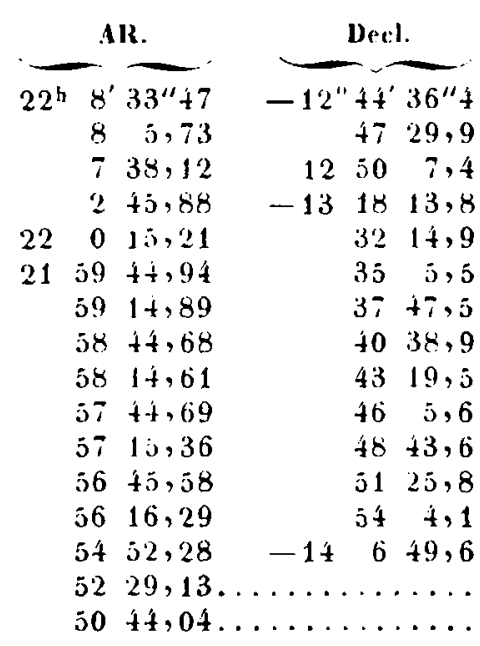

Osservazioni meridiane di Ebe.

$\begin{array}{rrrrrrrrrrr}\text { Agosto } & 5 & 13 & 12 & 49,7 & 22 & 17 & 42,15 & -12 & 47 & 51,7 \\ & 6 & 13 & 8 & 26,4 & & 17 & 12,25 & 13 & 2 & 10,3 \\ 7 & 13 & 4 & 1,8 & & 16 & 41,05 & 13 & 16 & 35,2 \\ 22 & 11 & 55 & 38,9 & & 6 & 54,11 & 17 & 6 & 0,7 \\ 23 & 11 & 50 & 59,8 & & 6 & 10,46 & 17 & 21 & 26,7 \\ 25 & 11 & 41 & 40,9 & & 4 & 42,68 & 17 & 52 & 4,8 \\ 26 & 11 & 37 & 1,4 & & 3 & 58,77 & 18 & 7 & 12,8 \\ 27 & 11 & 32 & 21,9 & & 3 & 15,18 & 18 & 22 & 13,1 \\ 28 & 11 & 27 & 42,4 & & 2 & 31,74 & 18 & 37 & 4,6 \\ & 29 & 11 & 23 & 3,0 & & 1 & 48,37 & 18 & 51 & 49,0 \\ \text { Settembre } & 30 & 11 & 18 & 24,7 & 22 & 1 & 5,59 & 19 & 6 & 26,0 \\ & 5 & 10 & 50 & 39,8 & 21 & 57 & 1,08 & 20 & 29 & 32,8 \\ & 11 & 10 & 23 & 24,7 & 21 & 53 & 30,00 & -21 & 43 & 36,2\end{array}$

Osservazioni meridiane di Vesta.

$\begin{array}{rr}\text { Agosto } & 24 \\ & 25 \\ 26 \\ 27 \\ 28 \\ 29 \\ 30 \\ \text { Settembre } \quad 4 \\ \\ 5 \\ 7\end{array}$

$\begin{array}{ccccc}\text { Settembre } & 11 & 11 & 44 & 24,7 \\ & 17 & 11 & 16 & 22,0 \\ & 22 & 10 & 52 & 58,8 \\ & 23 & 10 & 49 & 18,4 \\ 26 & 1034 & 19,8 \\ 14 & 1130 & 23,5\end{array}$

\begin{tabular}{|c|c|c|c|c|c|}
\hline 21 & 31 & 3,46 & -23 & 20 & 10,0 \\
\hline & 30 & 11,82 & 23 & 25 & 48,1 \\
\hline & 29 & 20,68 & 23 & 31 & 13,7 \\
\hline & 28 & 30,95 & 23 & 36 & 25,4 \\
\hline & 27 & 41,93 & 23 & 41 & 29,4 \\
\hline & 26 & 54,12 & 23 & 46 & 17,3 \\
\hline & 26 & 7,21 & 23 & 50 & 35,4 \\
\hline & 22 & 32,08 & 24 & 10 & 45,9 \\
\hline & 21 & 53,45 & 24 & 13 & 59,0 \\
\hline & 20 & 39,84 & -24 & 20 & 1,0 \\
\hline
\end{tabular}

Pallade.

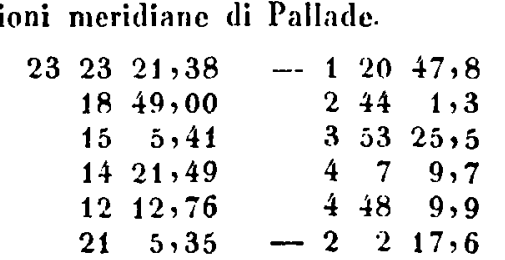

Lembo a cui fu collinato superiore inferiore

s. i. s. i.

s.

i.

s.

i.

s.

i.

s.

s.

Le osservazioni di Ebe sono corrette dell' aberrazione e parallasse.
Le osservazioni di Giove sono corrette dell'aberrazione e parallasse.
Le osservazioni di Vesta sono corrette dell' aberrazione e parallasse.


Nr. 990.

Osservazioni estrameridiane di Eunomia.

\begin{tabular}{|c|c|c|c|c|c|c|c|c|}
\hline 1855 & J. M. di Padova & $\mathbf{A R}$. & Decl. & $\begin{array}{c}\text { Nr. } \\
\text { dei confronti }\end{array}$ & $\begin{array}{l}\text { Posiz.app.d } \\
\text { AR. }\end{array}$ & ella di c & $\begin{array}{l}\text { confronto } \\
\text { Decl. }\end{array}$ & Autorità \\
\hline ggio17 & $12^{\mathrm{h}} 27^{\prime} 59^{\prime \prime} 7$ & $16^{\mathrm{b}} 56^{\prime} 43^{\prime \prime} 26$ & $-34^{\circ} 54^{\prime} 27^{\prime \prime} \overline{0}$ & 1 & $17^{\text {h }} \quad 9^{\prime} \quad 6^{\prime \prime} 62$ & $-34^{\circ}$ & ${ }^{\circ} 49^{\prime} 33^{\prime \prime} 5$ & 5825 \\
\hline 19 & 122154,3 & $\begin{array}{ll}16 & 54 \quad 51,80\end{array}$ & $-3451 \quad 2,0$ & 6 & $\begin{array}{lll}16 & 58 & 38,65\end{array}$ & -34 & 4839,8 & Da 4 confronti colla $\mathrm{p}$ \\
\hline 23 & 115045,5 & $16 \quad 50 \quad 59,04$ & $-344214,1$ & 3 & eadem & - & $-\quad-$ & --- \\
\hline Giugno 7 & 11020,6 & $1635 \quad 18,83$ & $-334737,1$ & 2 & $16 \quad 40 \quad 49,34$ & -.34 & 139,2 & 5632 B. A. \\
\hline
\end{tabular}

Le osservazioni di Eunomia non sono corrette nè dell'aberrazione, nè della parallasse.

Padova, 1. Ottobre 1855.

Virgilio Trettenero.

Originalbeobachtungen des Cometen von 1833 auf der Sternwarte zu Paramatta.

\section{( Fortsetzung.)}

Oct. 9. The comet is south following $\delta$ Scorpii. The star describing a chord North and the comet a chord South of the centre of the circle. - The night clouds with very high wind.

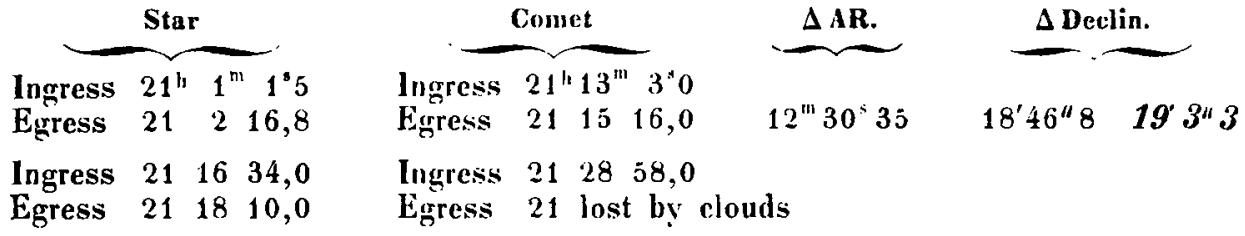

Clock last $15^{\circ}$.

Middle of observations in sidereal time $21^{\mathrm{h}} 13^{\mathrm{m}} 55^{\mathrm{s}} . \quad \Delta A R \cdot 12^{\mathrm{m}} 30^{5} 35 . \quad \Delta$ Decl. $18^{\prime} 46^{\mathrm{H}} 8 \quad 193^{\mathrm{u}} 3$.

Oct. 10. The night overcast, - no observations. -

Oct. 11. The Comet is North following $5 g$ Ophiuchi, both the star and comet describing a chord South of the centre of the circle. Star the most (more) southerly. Sky hazy in the west.

\begin{tabular}{|c|c|c|c|c|c|}
\hline & Star & & Comet & $\Delta \mathrm{AR}$ & $\Delta$ Decl. \\
\hline $\begin{array}{l}\text { Ingress } \\
\text { Egress }\end{array}$ & $\begin{array}{l}20^{\mathrm{ln}} 42^{\mathrm{m}} 16^{\mathrm{s}} 3 \\
20 \quad 43 \quad 59,3\end{array}$ & $\begin{array}{l}\text { lngress } \\
\text { Egress }\end{array}$ & $\begin{array}{l}20^{\mathrm{h}} 46^{\mathrm{m}} 31^{\mathrm{s}} 0 \\
20 \quad 48 \quad 35,5\end{array}$ & $4^{m} 25^{-5} 45$ & $3^{\prime} \quad 3^{\prime \prime} 0$ \\
\hline $\begin{array}{l}\text { Ingress } \\
\text { Egress }\end{array}$ & $\begin{array}{rrr}20 & 50 & 5,5 \\
20 & 51 & 21,0\end{array}$ & $\begin{array}{l}\text { Ingress } \\
\text { Egress }\end{array}$ & $\begin{array}{rrrr}20 & 54 & 25,0 \\
20 & 56 & 7,0\end{array}$ & $432,7 j$ & 230,5 \\
\hline $\begin{array}{l}\text { Ingress } \\
\text { Egress }\end{array}$ & $\begin{array}{lll}20 & 57 & 24,0 \\
20 & 58 & 49,4\end{array}$ & $\begin{array}{l}\text { Ingress } \\
\text { Egress }\end{array}$ & $\begin{array}{lll}21 & 1 & 33,0 \\
21 & 3 & 32,0\end{array}$ & 425,8 & 233,4 \\
\hline $\begin{array}{l}\text { Ingress } \\
\text { Egress }\end{array}$ & $\begin{array}{rrr}21 & 4 & 27,0 \\
21 & 6 & 2,0\end{array}$ & $\begin{array}{l}\text { Ingress } \\
\text { Egress }\end{array}$ & $\begin{array}{rrr}21 & 8 & 45,0 \\
21 & 10 & 46,0\end{array}$ & 431,0 & 340,6 \\
\hline $\begin{array}{l}\text { Ingress } \\
\text { Egress }\end{array}$ & $\begin{array}{rrr}21 & 12 & 5,0 \\
21 & 13 & 41,6\end{array}$ & $\begin{array}{l}\text { Ingress } \\
\text { Egress }\end{array}$ & $\begin{array}{lll}21 & 16 & 26,0 \\
21 & 18 & 27,0\end{array}$ & 433,2 & 330,4 \\
\hline $\begin{array}{l}\text { Ingress } \\
\text { Egress }\end{array}$ & $\begin{array}{rrr}21 & 19 & 18,0 \\
21 & 21 & 2,2\end{array}$ & $\begin{array}{l}\text { Ingress } \\
\text { Egress }\end{array}$ & $\begin{array}{lll}21 & 23 & 42,0 \\
21 & 25 & 48,0\end{array}$ & $\begin{array}{l}4 \quad 34,9 \quad 35,2\end{array}$ & 340,3 \\
\hline $\begin{array}{l}\text { Ingress } \\
\text { Egress }\end{array}$ & $\begin{array}{lll}21 & 27 & 11,6 \\
21 & 28 & 19,5\end{array}$ & $\begin{array}{l}\text { Ingress } \\
\text { Egress }\end{array}$ & $\begin{array}{llll}21 & 31 & 24,0 \\
21 & 33 & 25,0\end{array}$ & 438,95 & 414,6 \\
\hline
\end{tabular}

Clock fast $12^{\prime} 5$.

Middle of the observations in sidereal time $21^{\mathrm{t}} 9^{\mathrm{m}} 44^{\prime} 45^{\mathrm{s}}$. 